\title{
Sphingobacterium multivorum
}

National Cancer Institute

\section{Source}

National Cancer Institute. Sphingobacterium multivorum. NCI Thesaurus. Code C86959.

A species of aerobic, Gram negative, rod and cocci shaped bacteria assigned to the phylum Bacteroidetes. This species contains sphingolipids, can ferment glucose and is oxidase and urease positive. S. multivorum can be pathogenic in immunocompromised individuals. 